\section{PHILOSOPHICAL TRANSACTIONS A}

royalsocietypublishing.org/journal/rsta

\section{Research}

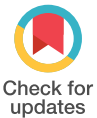

Cite this article: de Barros JA, Holik F, Krause

D. 2019 Indistinguishability and the origins of contextuality in physics. Phil. Trans. R. Soc. A

377: 20190150.

http://dx.doi.org/10.1098/rsta.2019.0150

Accepted: 10 May 2019

One contribution of 16 to a theme issue 'Contextuality and probability in quantum mechanics and beyond'.

\section{Subject Areas:}

quantum physics, set theory

\section{Keywords:}

contextuality, quasi-sets, quantum indistinguishability

\section{Author for correspondence:}

J. Acacio de Barros

e-mail: barros@sfsu.edu

\title{
Indistinguishability and the origins of contextuality in physics
}

\section{J. Acacio de Barros ${ }^{1}$, Federico Holik ${ }^{2}$ and}

Décio Krause ${ }^{3}$

'School of Humanities and Liberal Studies, San Francisco State University, San Francisco, CA, USA

${ }^{2}$ Universidad de La Plata/CONICET, La Plata, Argentina

${ }^{3}$ Department of Philosophy, Federal University of Brazil in Santa Catarina, Florianópolis, Santa Catarina, Brazil

\section{$J A B, 0000-0001-8233-2073$}

In this work, we discuss a formal way of dealing with the properties of contextual systems. Our approach is to assume that properties describing the same physical quantity, but belonging to different measurement contexts, are indistinguishable in a strong sense. To construct the formal theoretical structure, we develop a description using quasi-set theory, which is a settheoretical framework built to describe collections of elements that violate Leibnitz's principle of identity of indiscernibles. This framework allows us to consider a new ontology in order to study the properties of quantum systems.

This article is part of the theme issue 'Contextuality and probability in quantum mechanics and beyond'.

\section{Introduction}

The concept of a property of a quantum system is hard to define consistently. For instance, in a famous paper [1], Kochen \& Specker (KS) showed that attempting to assign truth values to a quantum property $[2,3]$, as predicted by the algebra of observables in a Hilbert space, may result in logical contradictions unless we assume that properties depend on which other properties are being simultaneously observed with it. This dependency is what is known in the literature as contextuality, reflecting the idea that properties are context-dependent-context being defined by the other simultaneously observed quantities. 
Quantum contextuality creates the difficulty that the value of a property becomes dependent on the observer's choice of what context to measure it in, i.e. with which other properties, and not with an intrinsic characteristic of the measured quantum system.

The contextual dependency of properties of quantum particles seems to be an essential aspect of the microscopic world. It is related not only to the KS apparent paradox mentioned above but also to Bell's proof of the incompatibility of quantum predictions with local-realism [4]. It is also an important resource in quantum computation [5], and perhaps the main reason why quantum computers outperform their classical counterparts [6]. Thus, it is not surprising that extensive research in contextuality has happened in recent decades, with entire conferences devoted to it (such as the Quantum Contextuality in Quantum Mechanics and Beyond workshop in Prague, or the Winer Memorial Lectures at Purdue University).

This paper examines contextuality in physics by extending a point of view put forth in references [7,8], namely that quantum indistinguishability is connected to contextuality. In [7], we argued that the indistinguishability of particles, expressed mathematically by a set-theoretical construct where the law of identity of indiscernibles is violated, invalidates the contradiction argument put forth by KS in [1] (see §2 for a sketch of the KS argument). In their paper, KS discuss the concept of quantum properties, represented by self-adjoint operators on a Hilbert space [9], and show that attempts to assign truth values to those properties in different experimental contexts fail if we assume that such properties are context-independent. We argued that KS argument was not necessarily valid for quantum systems because, since particles are in principle indistinguishable, it is not possible to say that we are talking about property $X$ or $Y$ of particle $A$ or particle B. All we know is that, in the case of A and B, we have two particles, and that they have different properties $X$ and $Y$, but we cannot, in principle, know which one has property $X$ or property $Y$.

Here, we extend the above notion to show that it is not just the indistinguishability of particles that may be at play in physics, but also the indistinguishability of properties, seen here as a representation of the conjunction of a specific measurement apparatus and a physical (quantum) system (e.g. a particle). In other words, as in the case of particles, we cannot know which property we are talking about. Two properties, A and A', may be indistinguishable, and this indistinguishability leads to apparent paradoxes if we treat them, as often is done in physics, as the same.

The idea of indistinguishability of properties can be seen as stemming from [10]. In that reference, it is argued that contextuality is about the identity of properties, in the sense that properties taken from different contexts must be considered different. In [10], properties are assumed to obey the classical theory of identity, formalized in it by the use of random variables in a probability space. The connection between identity and contextuality seems to also appear in the formalism of quantum mechanics. Take the case of three observables, such as Bell's case where observables $A$ and $A^{\prime}$ refer to Alice's observations and $B$ to Bob's. The self-adjoint operators in the subspace representing Bob's observable $B$ are the same when Alice measures $A$ or $A$, regardless of Alice's choice of measurement. It seems to be the same property, among the different contexts of measurement. The experimental set-up is the same as well: there is an operationally identifiable procedure that allows us to say that we are measuring $A$, on each instance. If we assume that all those instances represent the same property, we are led to contradictions. This situation leads the authors of [10] to conclude that properties associated with the same physical quantity, but considered in different contexts, cannot be the same. So, the distinction between $B$ in context $A$ and $B$ in context $A^{\prime}$ is that they are different properties, but not distinguishable. If they were distinguishable, i.e. if Bob could run an experiment where he could, just looking at $B$, determine Alice's choice of measuring $A$ or $\mathrm{A}^{\prime}$, they would be able to use this to signal to each other in a superluminal way. This signalling, of course, is forbidden by quantum mechanics [11].

How to make sense of the assertion that properties are different among different contexts, while it is, at the same time, the same quantity being measured? Reference [10] takes the approach of just using different random variables for each context. Concerning this point, a similar position is taken in $[12,13]$, where it is also assumed that properties associated with the 
same physical quantity, but considered in different contexts, even if the same mathematical object represents them, cannot be the same (and this is called extracontextuality). Though this is logically consistent, here we take an alternative stance: we will assume that properties-representing the same quantity to be measured-are indistinguishable among the different contexts, but yet, not the same. To do so, we use quasi-set theory [14], which is a logical formalism developed to deal with collections of truly indistinguishable entities. Quasi-sets have been applied to quantum mechanics before, in order to describe quantum non-individuality [15-17], and, as mentioned above, to avoid KS-type contradictions [7]. We see our approach as having two advantages over the more general one of [10]. First, it comes from a strong ontological assumption that quantum systems are truly, and in principle, non-distinguishable. This does not seem to be an epistemic issue, as there are real consequences for this indistinguishability at the level of particles (i.e. different quantum statistics, or other quantum effects such as Bose-Einstein condensates). The second advantage is that our approach stays closer to the Hilbert space representations of quantum theory by using the same random objects for all contexts.

This paper is organized as follows. For each section, we try to provide an intuitive and simplified version of the concepts discussed and then present them more formally. Our goal is to make this paper more accessible to a broader audience who may not be familiar with some of the ideas used here. In $\$ 2$, we discuss the concept of properties for quantum systems and show how they are problematic because of contextuality. In $\S 3$, we show how we can represent the indistinguishability of particles using the formalism of quasi-sets, constructed formally as an axiomatic theory where Leibniz's principle of identity is violated. Then, in $\S 4$, we extend the ideas of $\S 3$ to properties, and we show how, when properties are indistinguishable, the usual contextual inequalities are not derivable. We end the paper with some conclusions and final remarks in $\S 5$.

\section{Describing quantum systems}

To discuss the quantum case, let us start with the general concept of properties. ${ }^{1}$ Intuitively, a property is a characteristic or quality of something. For example, when we say that the sky is blue', the colour blue is a characteristic of how we perceive the sky. In physics, when we talk about the properties of a system, we mean something similar: what features this system has. For instance, if we say that a metal block is $32 \mathrm{~cm}$ long or $400 \mathrm{~g}$ in mass, this statement represents a property the block has: the property of being $32 \mathrm{~cm}$ long and having a mass of $400 \mathrm{~g}$. This concept of property is straightforward in classical physics, where we can talk about the volumes of solids, the temperature of an object or the energy of a system, to mention a few.

The most basic type of property is a binary property, i.e. a quality of the system that is either true or false. In other words, we can probe whether the system has or does not have the property. Some simple examples of binary properties are represented in the following questions: 'Does Federico have the property of being tall?' or 'Is it cold today?' Of course, such casual properties are not what we are talking about in physics, and we need to be more precise, going beyond defining which height we think is the minimum for being considered tall, or what temperature below which we feel it is cold. In physics, we need to talk about more complicated properties that can account for more specificity, such as today's temperature in degrees Celsius (it is $13^{\circ} \mathrm{C}$ outside, reads someone's thermometer) instead of merely saying it is cold or not.

It is straightforward to see that even such complicated properties are made up of several binary properties. Let us examine the temperature example. To measure the temperature means to give a number that is within the range of the thermometer (say -20 to $60^{\circ} \mathrm{C}$ ) and that is consistent with its precision $\left( \pm 0.5^{\circ} \mathrm{C}\right)$. Consider the following series of statements that can be either true or false. $A_{-20}=$ 'the tip of the mercury column of the thermometer is in the interval-20.0 $\pm 0.5^{\prime}$, $A_{-19}=$ 'the tip of the mercury column of the thermometer is in the interval $-19.0 \pm 0.5^{\prime}, A_{-18}=$ 'the tip of the mercury column of the thermometer is in the interval-18.0 \pm 0.5 ', and so on until

\footnotetext{
${ }^{1}$ We shall not give a detailed theory of properties relevant to the empirical sciences, and the interested reader may find references such as [18-20] or [21] as useful and comprehensive resources; see also [22-24] for the notion of property in the quantum logical approach, which plays a key role in quantum mechanics and the derivation of the KS theorem.
} 
$A_{60}=$ 'the tip of the mercury column of the thermometer is in the interval $60.0 \pm 0.5$ '. Each of those statements is compatible (i.e. one can verify the veracity of each of those questions simultaneously), and they are complementary (i.e. one and only one of them may be true at a given time). So, the statement 'it is $13^{\circ} \mathrm{C}$ outside' means that the proposition $A_{13}=$ 'the tip of the mercury column of the thermometer is in the interval $13.0 \pm 0.5^{\prime}$ is true, whereas all other complementary propositions $A_{i}, i \neq 13$, are false. Any numerical property could be represented this way, as made up of several individual and compatible binary properties. So, properties such as mass, charge, position, temperature, entropy, and length can be though as a combination of a large (sometimes infinite) number of binary properties where only one of them can be true at a time.

There is a fascinating connection between properties and ontology in classical physics. In classical Newtonian physics, physical systems are composed of particles, whose fundamental properties are their mass, position and velocity. Because mass is a constant for Newtonian particles, only position and velocity can vary, and the value of all particles' positions and momenta are called the state of the system. Any other property, such as the system's energy, temperature or length (if talking about a solid made of particles itself), is definable in terms of the properties of the fundamental constituents of the system, namely the position and velocity of the particles. Furthermore, given a system of particles and their interactions, their present state, i.e. their position and velocity, determines their future state, and, therefore, any properties associated with the system. But, more importantly, given that properties are definable in terms of two quantities that are simultaneously measurable, position and velocity, it follows that each property can be thought as a subset of the space of all possible positions and velocities (usually $\mathbb{R}^{6 N}$, where $N$ is the number of particles and 6 the number of components of the position and velocity vectors necessary to describe the particle). Therefore, if we wish to define an algebra of properties, this algebra would be simply a Borel algebra on $\mathbb{R}^{6 N}$.

In quantum theory, things are very different. First, there is no simple and widely accepted ontology for quantum systems similar to the classical one. ${ }^{2}$ Second, the state of a quantum system is not definable in terms of the position and velocity of its particles. The reason is a fundamental one: contrary to classical particles, position and velocity of a quantum particle cannot be, in principle, measured simultaneously with as much precision as we wish. Therefore, the idea of defining properties as subsets of $\mathbb{R}^{6 N}$ is not a straightforward matter. ${ }^{3}$

Instead, binary properties are represented in quantum theory by projection operators in a separable Hilbert space, $\mathcal{H}[17,22]$. The Hilbert space itself is determined by the number of such binary properties that we can maximally observables. More complex outcomes of experiments and their associated properties are modelled by self-adjoint operators in $\mathcal{H}$. Owing to the spectral decomposition theorem, Hermitian operators can always be written as sums of projection operators. In other words, we can think of Hermitian operators as made up of several binary properties, which, due to their connections to experiments, are called observables. Thus, observables and the Hilbert space are dependent not only on the system but on our ability to extract information from this system. The more information, the larger the Hilbert space becomes.

To give a less abstract example, take the case of a single electron. If we were only able to measure its position in the $x$-direction, its Hilbert space would be $\mathcal{L}^{2}$, the space of all squareintegrable functions, and a vector in this space would be a function $\psi \in \mathcal{L}^{2}$ whose absolute value squared, $|\psi(x)|^{2}$, at $x$ gives the probability density that the electron is found between $x$ and $x+d x$ if a measurement of position is performed. In this Hilbert space, the position operator is simply $x$. Correspondingly, the observable associated with the property 'momentum' is the operator $i \hbar \partial / \partial x$

\footnotetext{
${ }^{2}$ It is important to mention that Bohm's theory [25] provides an ontology close to a classical one, but it is far from being widely accepted among physicists [26]. One of the main reasons for this, is, perhaps, that its hidden-variables behave in a manifestly non-local way. Furthermore, the hidden-variables introduced are not of much use in practice, given that they cannot be manipulated in the laboratory (due to its hidden character). Thus, they play only an ad hoc explanatory role, without giving place to any relevant predictions. Our approach in this work aims to stay closer to most physicists guiding intuitions in their practice.
}

${ }^{3}$ Attempts to do so lead to quasi-probability distributions [27]. 
on $\mathcal{L}^{2}$. However, electrons have another property of interest: spin. The Hilbert space for spin $1 / 2$, as is the case for the electron, is $\mathbb{C}^{2}$. So, if we were to measure only position or momentum, the Hilbert space would be $\mathcal{L}^{2}$; if we were to measure only spin (regardless of direction), the Hilbert space would be $\mathbb{C}^{2}$; and if we were to measure both position and spin, the Hilbert space would be $\mathcal{L}^{2} \otimes \mathbb{C}^{2}$. Things get more complicated as we increase the number of particles, or if we increase the number of possible observables.

As mentioned above, properties related to a Hilbert space through observable operators. Given a quantum system $S$, we can construct a Hilbert space $\mathcal{H}$, whose basis represents a set of possible projection operators that completely span $\mathcal{H}$. This set of projection operators provide a maximal set of compatible properties of $S$. We can then use those properties to create more complex properties in the form of Hermitian operators.

Again, let us explore this with a simple example: a three-dimensional Hilbert space, $\mathbb{C}^{3}$. Since this space is three-dimensional, it follows that a basis for this space is constituted of three linearly independent vectors, say

$$
\mathbf{e}_{1}=\left(\begin{array}{l}
1 \\
0 \\
0
\end{array}\right), \quad \mathbf{e}_{2}=\left(\begin{array}{l}
0 \\
1 \\
0
\end{array}\right) \quad \text { and } \quad \mathbf{e}_{3}=\left(\begin{array}{l}
0 \\
0 \\
1
\end{array}\right)
$$

and the corresponding projectors associated with each vectors are

$$
P_{\mathbf{e}_{1}}=\left(\begin{array}{ccc}
1 & 0 & 0 \\
0 & 0 & 0 \\
0 & 0 & 0
\end{array}\right), \quad P_{\mathbf{e}_{2}}=\left(\begin{array}{ccc}
0 & 0 & 0 \\
0 & 1 & 0 \\
0 & 0 & 0
\end{array}\right) \quad \text { and } \quad P_{\mathbf{e}_{3}}=\left(\begin{array}{ccc}
0 & 0 & 0 \\
0 & 0 & 0 \\
0 & 0 & 1
\end{array}\right) .
$$

We can see that $P_{\mathbf{e}_{1}}+P_{\mathbf{e}_{2}}+P_{\mathbf{e}_{3}}=\hat{1}$, where $\hat{1}$ is the identity matrix, which is a consequence of $\mathbf{e}_{1}$, $\mathbf{e}_{2}$ and $\mathbf{e}_{3}$ forming a basis for $\mathcal{H}$. It is easy to create now, in this formalism, an observable that is associated with the property of having values 1,2 or 3 as merely $P_{\mathbf{e}_{1}}+2 P_{\mathbf{e}_{2}}+3 P_{\mathbf{e}_{3}}$.

Once one has a basis for $\mathcal{H}$, one can define observables as above. However, it is always possible to define another basis. In quantum theory, this new basis will correspond to new observable properties. What is important here is that a property with a definite value on one basis may not be associated with a state that has definite values for another property (represented by another basis). Furthermore, once we measure the observable associated with the new basis, and find out a value for a given property, the new state of the system will be associated with this property, and the old basis (and their corresponding properties) will not have definite values anymore. An observation (or measurement) affects the state of the system.

In other words, the sequential observation of properties on different basis may lead to changes in the outcomes of past observations. Properties become dependent on the how we observe them: if we first observe A and then B, we may get something different from observing $B$ and then $A$. Even more importantly, if we observe A, B, and then A, the second time we observe A its value may be different. Additionally, attempts to assign values to properties that are independent of how we observe them will lead to inconsistencies.

The above argument is at the core of KS's theorem [1]. In an example provided by Cabello et al. [28], we start with a specific set of projection operators $P_{i}, i=1, \ldots, 18$, in a Hilbert space of dimension four. The set $\left\{P_{i}\right\}$ is selected such that there are nine contexts such that the sum of the four $P_{i}^{\prime}$ 's in it is the identity operator (e.g., $P_{1}+P_{2}+P_{3}+P_{4}=\hat{1}$ ). Furthermore, the contexts are selected such that each projector appears twice, once in two different contexts (e.g., $P_{1}+P_{2}+P_{3}+$ $P_{4}=\hat{1}$ and $\left.P_{1}+P_{5}+P_{6}+P_{7}=\hat{1}\right)$. The consequence is that we have nine equations, one for each context, where they all add to $\hat{1}$, and such that each operator appears twice. The contradiction comes from associating each projector with the same value 1 (true) or 0 (false) in all contexts. The sum of all projectors' values on the nine equations adds to an even number (each appears twice). However, since each equation adds to one on their right-hand side, their sum needs to add to nine, which is an odd number, and we reach a contradiction. This argument is the essence 
of the KS theorem: the assumption that the values of $P_{i}$ are independent of context leads to a contradiction.

One may object that the above arguments were focused solely on cases where the property of the system is known with certainty, i.e. one can assign to it a truth value. One may argue that in quantum physics, properties are not deterministic, and one must talk about probabilities, which would make the above arguments not apply. However, this is not the case. It is possible to show that the underlying assumption that properties have a value, even though we may not know what they are and represent them with a probability function, is incompatible with quantum theory [29]. The reason is that standard probability theory assumes an underlying consistency through a Boolean algebra.

To accommodate the quantum predictions, one must either expand the number of properties to include other properties that are co-measured (see [30] and references therein), or one needs to modify probability theory, either by allowing them to be less than zero [31-33], by changing the rule for adding probabilities [34,35], by modifying the algebra of events [23,36,37], or by rethinking about measurement outputs as depending on all components of an examined experiment [38]. However, regardless of how we choose to deal with such issues, the critical aspect of the quantum world is that quantum properties are not definable in a consistent way if we require classical logic and context independence. This quantum contextuality is essential for any ontology associated with it, and we will explore it in more detail in $\S 4$.

\section{Indistinguishability of particles}

Two remarkable features characterize compound quantum systems. One is entanglement, which can be interpreted as the impossibility of describing specific quantum correlations by appealing to mixtures of classical correlations [39]. The other feature-the one that we are interested in-is indistinguishability: when quantum systems of the same kind are put together, they display statistics which are very different from those which are used to describe distinguishable entities. ${ }^{4}$ Indistinguishability is reflected in the symmetrization postulate and the celebrated Bose and Fermi statistics. This feature lies behind very important fields of research, such as the study of Bose-Einstein condensates. In recent years, the difference between entanglement and indistinguishability has been studied in detail: it turns out that these are very different physical features, in the sense that a quantum system can be prepared in a fully symmetrized state in which no non-local correlations are present [41]. This distinction leads us to the question of whether it is possible to consider quantum indistinguishability as a resource. What is the relationship, if any, between quantum indistinguishability and contextuality? In order to explore possible answers for these questions in the following sections, let us first review the formalism for indistinguishable particles.

Let us illustrate how the formalism works for only two fermions. For this case, if we know that one particle is in state $|b\rangle$ and the other in state $|a\rangle$, then, using the symmetrization postulate, the joint state will be given by

$$
|\psi\rangle=\frac{1}{\sqrt{2}}(|a\rangle \otimes|b\rangle-|b\rangle \otimes|a\rangle)
$$

The above state means that there is one particle in each state. However, the symmetrization tells us that we cannot tell which one is which: a permutation of the particles yields an overall minus sign, and, thus, the probabilistic predictions of the theory are the same. This situation leads many authors-including Schrödinger-to conclude that quantum systems, in certain situations, cannot be considered as individuals [42].

\footnotetext{
${ }^{4}$ We emphasize that the indistinguishability of particles is an ontological assumption. For example, as mentioned before, in Bohmian theory $[25,40]$ the ontology is classical, with quantum effects originating from the quantum potential. The antisymmetrization or symmetrization of the wave function, in this theory, leads to non-local effects on the particles due to the quantum potential. Though the authors of this paper are sympathetic to Bohm's approach, we are also aware that the majority of the physics community rejects it, perhaps mainly because of its classical ontology. In this paper, we embrace the quantum weirdness and try to explain quantum effects based on a non-classical ontology of indistinguishable particles.
} 
In the position representation, the wave function associated with our two-particle state in (3.1) is given by

$$
\psi(x, y)=\frac{1}{\sqrt{2}}\left(\psi_{a}(x) \otimes \psi_{b}(y)-\psi_{b}(x) \otimes \psi_{a}(y)\right)
$$

where $x$ and $y$ are the coordinates of particles 1 and 2. When $x \longrightarrow y$, i.e. when the particles are close to each other, we observe that the square modulus of the wave function

$$
|\psi(x, y)|^{2}=\frac{1}{2}\left(\left|\psi_{a}(x) \psi_{b}(y)\right|^{2}+\left|\psi_{b}(x) \psi_{a}(y)\right|^{2}-2 \Re\left(\psi_{a}(x) \psi_{b}(y) \psi_{b}^{\star}(x) \psi_{a}^{\star}(y)\right)\right),
$$

tends to zero. This implies that no two fermions can be found occupying the same state. If the wave functions $\left|\psi_{a}(x)\right|$ and $\left|\psi_{b}(y)\right|^{2}$ have compact support, we see that there are no indistinguishability effects when $|x-y|$ is big enough. Thus, when the particles get close each other, we can go continuously from a distinguishability for all practical purposes situation, to a non-distinguishability one.

The state of a Bosonic system is similar to (3.1), but there is ' + ' sign instead of a ' - .' In the first case, the states are symmetric under the permutation of particles, while in the second, anti-symmetric. This symmetry for Bosonic systems changes the statistics considerably: unlike Fermions, it is possible to have an arbitrary number of Bosons occupying the same state.

In order to study the case with arbitrary particle number, it is useful to consider the Fock-space formalism. The standard Fock-space is built up from the one-particle Hilbert space as follows. Let $\mathcal{H}$ be a Hilbert space and define

$$
\begin{aligned}
& \mathcal{H}^{0}=\mathbb{C}, \\
& \mathcal{H}^{1}=\mathcal{H}, \\
& \mathcal{H}^{2}=\mathcal{H} \otimes \mathcal{H}, \\
& \vdots \\
& \mathcal{H}^{n}=\mathcal{H} \otimes \cdots \otimes \mathcal{H} .
\end{aligned}
$$

The Fock-space is thus constructed as the direct sum of $n$ particle Hilbert spaces,

$$
\mathcal{F}=\bigoplus_{n=0}^{\infty} \mathcal{H}^{n}
$$

When dealing with bosons or fermions, the symmetrization postulate must be imposed. Thus, given a vector $v=v_{1} \otimes \cdots \otimes v_{n} \in \mathcal{H}^{n}$, define

$$
\sigma^{n}(v)=\left(\frac{1}{n !}\right) \sum_{P} P\left(v_{1} \otimes \cdots \otimes v_{n}\right)
$$

and

$$
\tau^{n}(v)=\left(\frac{1}{n !}\right) \sum_{P} s^{p} P\left(v_{1} \otimes \cdots \otimes v_{n}\right),
$$

where

$$
s^{p}= \begin{cases}1 & \text { if } \mathrm{p} \text { is even } \\ -1 & \text { if } \mathrm{p} \text { is odd }\end{cases}
$$

Let

$$
\mathcal{H}_{\sigma}^{n}=\left\{\sigma^{n}(v): v \in \mathcal{H}^{n}\right\}
$$

and

$$
\mathcal{H}_{\tau}^{n}=\left\{\tau^{n}(v): v \in \mathcal{H}^{n}\right\}
$$

Thus, we have the Fock-space

$$
\mathcal{F}_{\sigma}=\bigoplus_{n=0}^{\infty} \mathcal{H}_{\sigma}^{n}
$$


for bosons and

$$
\mathcal{F}_{\tau}=\bigoplus_{n=0}^{\infty} \mathcal{H}_{\tau}^{n}
$$

for fermions.

The standard wave mechanics approach to the description of multi-particle systems uses the kinetic energy operator

$$
T_{n}=\sum_{i=1}^{n} T_{1}\left(r_{i}\right)
$$

for $n$ particles, where $T_{1}(r)=-\left(\left(\hbar^{2} \nabla^{2} / 2 m\right)\right)$. A similar expression holds for the external potential. For a pairwise interaction potential, we have

$$
V_{n}=\sum_{i>j=1}^{n} V_{2}\left(\mathbf{r}_{i}, \mathbf{r}_{j}\right)
$$

The total Hamiltonian operator is thus given by

$$
H_{n}=\sum_{i=1}^{n}\left[\left(-\frac{\hbar^{2} \nabla_{i}^{2}}{2 m}\right)+V_{1}\left(\mathbf{r}_{i}\right)+\sum_{i>j=1}^{n} V_{2}\left(\mathbf{r}_{i}, \mathbf{r}_{j}\right)\right] .
$$

The $n$-particles wave function can be expressed as

$$
\Psi_{n}\left(\mathbf{r}_{1}, \ldots, \mathbf{r}_{n}, t\right)
$$

and it is a solution of the Schrödinger's equation

$$
H_{n} \Psi_{n}=i \hbar \frac{\partial}{\partial t} \Psi_{n}
$$

\section{Indistinguishability of properties}

We now move to discuss what we call the indistinguishability of properties, starting with the concept of properties. Suppose that we have the quantum system formed by parts $S_{1}$ and $S_{2}$. The observables associated with the compound system will be represented by the algebra $\mathcal{B}(\mathcal{H})$, with $\mathcal{H}=\mathcal{H}_{1} \otimes \mathcal{H}_{2}$. For any observable $A$ of $S_{1}$, we may consider the observable of the compound system $A \otimes B$. The interpretation of $A \otimes B$ is that we measure $A$ in $S_{1}$ and $B$ in $S_{2}$. However, for each $A \in \mathcal{B}\left(\mathcal{H}_{1}\right)$, there are infinitely many possible observables to chose in $\mathcal{B}\left(\mathcal{H}_{2}\right)$. Each one of these possibilities, defines a different measurement context. Thus, we can define the set

$$
C_{A}=\left\{A \otimes B \mid B \in \mathcal{B}\left(\mathcal{H}_{2}\right)\right\},
$$

which enables us to represent all possible contexts associated with a single observable $A \in \mathcal{B}\left(\mathcal{H}_{1}\right)$. Now, prepare $N$ copies of the compound system in the same state $\rho$ and measure only the observable $A$ of $S_{1}$. The state of $S_{1}$ is given by $\rho_{1}=\operatorname{tr}_{2}(\rho)$. From the preparation point of view, all these processes are indistinguishable: we prepare $N$ copies of the compound system and measure the same observable $A$ of $S_{1}$. However, even if these measurements are indistinguishable for an observer focused only on $S_{1}$, they are not the same: a measurement of $A$ in $S_{1}$ could be performed jointly with an arbitrary element of $C_{A}$, and these elements could change on each run of the experiment. As an example, if $N=N_{1}+N_{2}$, we can perform the experiment $A \otimes B N_{1}$ times, and $N_{2}$ times the experiment $A \otimes B^{\prime}$ (for $B \neq B^{\prime}$ ). Each context defined on the compound system gives us a different observable on the compound system, but an indistinguishable one for system $S_{1}$. Thus, for the observer focused on subsystem $S_{1}$, a measurement of the property associated with the physical quantity $\mathcal{A}$ can be represented by a collection of indistinguishable but yet different observables.

There is a formalism that allows dealing properly with collections of truly indiscernible entities, namely, quasi-set theory (for details, see for example [14-17]). In this theory, there is a 
primitive notion of indistinguishability, represented by the symbol ' $\equiv$ '. The axioms are written in such a way that $X \equiv Y$ does not necessarily imply $X=Y$. The equality symbol ' $=$ ' can only be applied to special elements of the theory (i.e., the classical ones). Thus, we can represent the observable quantity $\mathcal{A}$ by a quasi-set, which we call $[A]$, formed by the collection of all possible experimental contexts associated with $\mathcal{A}$. The elements of $[A]$ are all indistinguishable, but not the same (i.e. the quasi-cardinal-which represents the number of elements-of $[A]$ is greater than 1), in the sense that $X \equiv Y$, for all $X, Y \in[A]$. What are the valuations compatible with this description? We must be careful. Using quasi-set theory, if we try to assign a value to each element of $[A]$, we define a quasi-function $f:[A] \longrightarrow\{0,1, \ldots, d-1\}$ (here, we are assuming that $\mathcal{A}$ defines a $d$-dimensional observable). However, unlike the classical case, where we have $d^{\sharp[A]}$ valuations, there are only $d$ quasi-functions of this kind, namely $\langle[A], 0\rangle,\langle[A], 1\rangle, \ldots,\langle[A], d-1\rangle$, because all ordered pairs collapse into the same class. This means that, considered as an observable, the only values that $A$ can take are given by $\{0,1, \ldots, d-1\}$. This is compatible with what we observe in an actual experiment: the outcome set is given by $\{0,1, \ldots, d-1\}$. However, something more interesting happens when we try to assign values to the elements of $[A]$ previous to measurement. Let us do this as follows. In order to pick up one element of $[A]$, consider a strong singleton $[[X]] \subseteq[A]$. In quasi-set notation, this means that $\mathrm{qc}([[X]])=1$ and $\forall x \in[[X]]$, we have $x \in[A]$. Now, take $j \in\{0,1, \ldots, d-1\}$ and form the ordered pair $\langle[[X]] ; j\rangle$ in such a way that the pair has only two elements (this can be done in quasi-set theory). This pair can be interpreted as follows: assign the value $j$ to the context $[[X]]$. All contexts are indistinguishable from the perspective of the observer associated with $S_{1}$ : if the rest of the universe is ignored, each representative of class $[A]$ is indistinguishable from the others. However, they are different for the joint system since the different contexts define distinguishable global observables. By forming pairs of the form $\langle[[X]] ; j\rangle$, a definite value can be assigned to an observable in a given context. But an indistinguishable observable taken from a different context may have a different value. In this way, we see how a kind of logical indistinguishability, one taken from quasi-set theory, can be used to model quantum contextuality suitably.

In order to illustrate the description of contextuality using quasi-set theory with more detail, let us consider first a classical system formed of three dichotomic random variables $X, Y$ and $Z$, having values in the set $\{-1,1\}$. Let us assume that $X, Y$ and $Z$ obey the classical theory of identity (and, accordingly, that these variables retain their identity among the different contexts in which they may appear). Thus, for example, we are assuming that $X$ is the same, independently of whether it is measured in connection with $Y$ or in connection with $Z$. A similar consideration holds for $Y$ and $Z$. In this way, we obtain the following (classical) table:

\begin{tabular}{c|c|c|c|c|c}
$X$ & $Y$ & $Z$ & $X Y$ & $X Z$ & $Y Z$ \\
\hline 1 & 1 & 1 & 1 & 1 & 1 \\
1 & 1 & -1 & 1 & -1 & -1 \\
1 & -1 & 1 & -1 & 1 & -1 \\
-1 & 1 & 1 & -1 & -1 & 1 \\
1 & -1 & -1 & -1 & -1 & 1 \\
-1 & -1 & 1 & 1 & -1 & -1 \\
-1 & 1 & -1 & -1 & 1 & -1 \\
-1 & -1 & -1 & 1 & 1 & 1
\end{tabular}

Using the above table, one can verify that the values of the compound random variables $X Y, X Z$ and $Y Z$ satisfy the inequality

$$
-1 \leq X Y+X Z+Y Z \leq 3
$$

Thus, by convexity, their mean values $\langle X Y\rangle,\langle X Z\rangle$ and $\langle Y Z\rangle$ must, in turn, satisfy

$$
-1 \leq\langle X Y\rangle+\langle X Z\rangle+\langle Y Z\rangle \leq 3
$$

Now, let us see what happens if, instead of assuming a classical theory of identity, we consider that $X, Y$ and $Z$ define indistinguishable properties. Thus, the only thing we can say 
is that we have classes of indistinguishable properties $[X],[Y]$ and $[Z]$, formed by all possible indistinguishables from $X, Y$ and $Z$, respectively. Thus, when considering, for example, $X$ in connection with $Y$ and afterwards, in connection with $Z$, we will have $X$ and $X^{\prime} Z$, with $X^{\prime}$ indistinguishable from $X$ and $Z^{\prime}$ indistinguishable from $Z$-yet not the same! Thus, the value that we must assign to $X$ needs not to be the same as the one that we assign to $X$. Therefore, if we proceed as before and consider all possibilities, we don't have a definite value for $X$, but a collection of them: a value attached to each element of the class $[X]$, by appealing to quasipairs of the form $\langle[[X]],-1\rangle$ and $\langle[[X]], 1\rangle$ (being $[[X]] \subseteq[X]$ a strong singleton of $[X]$ ). A similar consideration holds for $Y$ and $Z$. The only thing we can do, regarding joint measurements, is to write down a table like

\begin{tabular}{c|c|c}
$X^{\prime} Y^{\prime}$ & $X^{\prime \prime} Z^{\prime}$ & $Y^{\prime \prime} Z^{\prime \prime}$ \\
\hline 1 & 1 & 1 \\
1 & -1 & -1 \\
-1 & 1 & -1 \\
-1 & -1 & 1 \\
-1 & -1 & 1 \\
1 & -1 & -1 \\
-1 & 1 & -1 \\
-1 & -1 & -1 \\
1 & 1 & -1 \\
1 & -1 & 1 \\
-1 & 1 & 1
\end{tabular}

where the primed quantities are indistinguishables from $X, Y$ and $Z$. Notice that the last four lines of the above table are strictly forbidden for classical random variables or, in our terminology, for random variables obeying the classical theory of identity. However, this implies that inequality (4.3) will be violated by random variables of this sort. Thus, we find that the violation of the theory of identity for properties can be used to describe contextuality naturally.

It is important to remark that this is not a quantum example. The reason is that, for three projection operators that commute pairwise, it follows that it is possible to define a set of vectors on the Hilbert space such that those vectors are eigenvectors of those projectors. Therefore, it follows that a joint probability distribution exists, and the properties associated with the projectors are not contextual. In principle, we could extend the argument to a Bell-type scenario with four properties if necessary (though it would be more cumbersome).

It is interesting to compare our approach with previous ones. The KS theorem has a very straightforward consequence: to assume that a given property possesses the same value among different contexts leads to contradictions. This has led many authors to conclude random variables-representing the same physical quantity-are different. In other words, that contexts can be used to index random variables in such a way that they become different (see, for example, [10]). According to the classical theory of identity, this sounds like a reasonable conclusion, but there remains a feeling that with such a proliferation of properties-in most examples of interest, there are, indeed, infinitely many contexts for each given propertya high ontological price is paid, especially considering that it is the same physical property among all possible contexts. Our framework is a way out of this situation since one can speak about indistinguishable properties in a strong sense but, at the same time, the instances of these properties are not forced to reach the same values on each context in which they are considered.

\section{Conclusion}

In this work, we proposed a formal framework for dealing with the properties of contextual systems. According to our proposal, properties describing the same physical quantity, but belonging to different measurement contexts, are not different, but neither are they equal. They 
are indistinguishable in a strong sense. The existence of such objects requires, mathematically, the introduction of a theory that allows a violation of Leibnitz's principle of identity of indiscernibles.

Quasi-set theory is such a theory, a set-theoretical framework that allows for the description of collections of entities that do not obey the classical principle identity $[14,42,43]$. Quasi-set theory includes objects that obey a weaker relationship of indistinguishability, allowing for entities being different solo numero. Here, we showed that quasi-sets could be used to describe quantum contextuality consistently. We believe this approach opens the door for a new ontology for describing quantum properties.

It is useful to compare our proposal with other ones. In the contextuality, by default approach $(\mathrm{CdB})$, properties belonging to different contexts are considered different $a b$ initio. If this is done, we can assume that not only properties obey the classical theory of identity, but they also satisfy classical probability theory. The cost of this approach is that properties then become context-dependent, and thus dependent on the observer's choice of context. Our framework is an alternative one that allows reconciling different values in different measurement contexts with the same property. In the other approaches using non-standard probability, it is possible to argue (and we did so in $\S 4$ ) that the use of indistinguishable objects from quasi-set leads to non-standard probabilities. This, in a sense, may provide an interpretation for the appearance of non-standard theories in quantum physics, and we believe it is a topic that should be investigated further. As such, it would also be interesting to study the consequences for probability theory of assuming random variables that do not obey the classical theory of identity. Such a non-standard probability calculus may be useful to understand the peculiar behaviour of probabilities in quantum theory. In this direction-and, in connection with the problem of identical particles-we hope that by addressing contextuality in quantum theory using quasi-sets, may help to understand the link between the underlying quantum particle ontology and their properties.

The ontology behind our approach assumes that properties are, in reality, associated with collections of indistinguishable entities. This ontology has exciting consequences for answering the following question: What are the necessary and sufficient conditions for contextuality? According to our proposal, contextuality appears whenever properties depart from classical identity theory. There could be many ways in which this may happen, quantum mechanics being a particular case. This opens the door for studying generalized probabilistic models using quasi-set theory or similar set-theoretical frameworks. In particular, it would be interesting to investigate which constraints in the indistinguishability of properties would produce quantum boundaries, such as Tsirelson's.

The main advantage of our approach is that it is a more natural description of what happens to properties in the quantum realm. The main disadvantage is that it is not as general as other proposals, such as contextuality by default, as it would not apply to non-quantum contextual systems since we expect such systems not to have an ontological issue with identity. However, perhaps more interesting for this paper's authors, is that our approach points toward a feasible quantum ontology—-perhaps a physical principle — that needs to be thought in more detail.

Data accessibility. This article has no additional data.

Authors' contributions. J.A.d.B. and F.H.H. wrote the main draft. All authors participated in the discussions.

Competing interests. We declare we have no competing interests.

Funding. No funding has been received for this article.

Acknowledgements. The authors thank Pawel Kurzinsky for discussions on the issue of contextuality and indistinguishability. We also benefited from conversations with participants at the Winer Memorial Lectures held at Purdue University in November 2018.

\section{References}

1. Kochen S, Specker EP. 1967 The problem of hidden variables in quantum mechanics. J. Math. Mech. 17, 59-87.

2. Piron C. 1976 On the Foundations of Quantum Physics. In Quantum Mechanics, Determinism, Causality, and Particles: An Int. Collection of Contributions in Honor of Louis de Broglie on the 
Occasion of the Jubilee of His Celebrated Thesis (eds M Flato, Z Maric, A Milojevic, D Sternheimer, JP Vigier). Mathematical Physics and Applied Mathematics. pp. 105-116. Dordrecht: Springer

The Netherlands.

3. von Neumann J. 1983 Mathematical foundations of quantum mechanics. Princeton, NJ: Princeton University Press. Translated by Robert T. Beyer from the 1932 German edition.

4. Bell JS. 1964 On the Einstein-Podolsky-Rosen paradox. Physics 1, 195-200. (doi:10.1103/ PhysicsPhysiqueFizika.1.195)

5. Veitch V, Ferrie C, Gross D, Emerson J. 2012 Negative quasi-probability as a resource for quantum computation. New J. Phys. 14, 113011. (doi:10.1088/1367-2630/14/11/113011)

6. Howard M, Wallman J, Veitch V, Emerson J. 2014 Contextuality supplies the 'magic' for quantum computation. Nature 510, 351-355. (doi:10.1038/nature13460)

7. de Barros JA, Holik F, Krause D. 2017 Contextuality and indistinguishability. Entropy 19, 435. (doi:10.3390/e19090435)

8. Kurzyñski P. 2017 Contextuality of identical particles. Phys. Rev. A 95, 012133. (doi:10.1103/ PhysRevA.95.012133)

9. von Neumann J. 1996 Mathematical foundations of quantum mechanics. Princeton, NJ: Princeton University Press.

10. Dzhafarov EN, Kujala JV. 2014 Contextuality is about identity of random variables. Phys. Scr. T163, 014009. (doi:10.1088/0031-8949/2014/T163/014009)

11. Dieks D. 1982 Communication by EPR devices. Phys. Lett. A 92, 271-272. (doi:10.1016/ 0375-9601(82)90084-6)

12. Auffèves A, Grangier P. 2016 Contexts, systems and modalities: a new ontology for quantum mechanics. Found. Phys. 46, 121-137. (doi:10.1007/s10701-015-9952-z)

13. Auffèves A, Grangier P. 2018 Extracontextuality and extravalence in quantum mechanics. Phil. Trans. R. Soc. A 376, 20170311. (doi:10.1098/rsta.2017.0311)

14. Krause D, French S. 1995 A formal framework for quantum non-individuality. Synthese 102, 195-214. (doi:10.1007/BF01063905)

15. Domenech G, Holik F. 2007 A discussion on particle number and quantum indistinguishability. Found. Phys. 37, 855-878. (doi:10.1007/s10701-007-9129-5)

16. Domenech G, Holik F, Krause D. 2008 Q-spaces and the foundations of quantum mechanics. Found. Phys. 38, 969-994. (doi:10.1007/s10701-008-9246-9)

17. Domenech G, Holik F, Massri C. 2010 A quantum logical and geometrical approach to the study of improper mixtures. J. Math. Phys. 51, 052108. (doi:10.1063/1.3429619)

18. Krantz D, Luce D, Suppes P, Tversky A. 1971 Foundations of measurement, vol. I: Additive and polynomial representations. Mineola, New York, NY: Dover Publications Inc.

19. Krantz DH, Suppes P, Duncan-Luce R, Tversky A. 1989 Foundations of Measurement, vol. II: Geometrical, Threshold, and Probabilistic Representations, vol. 2. Mineola, New York, NY: Dover Publications Inc.

20. Luce D, Krantz D, Suppes P, Tversky A. 1990 Foundations of measurement, vol. III: representation, axiomatization, and invariance. Mineola, New York, NY: Dover Publications Inc.

21. Suppes P. 2002 Representation and invariance of scientific structures. Stanford, California: CSLI Publications.

22. Birkhoff G, von Neumann J. 1936 The logic of quantum mechanics. Ann. Math. 37, 823-843. (doi:10.2307/1968621)

23. Foulis DJ. 1999 A half-century of quantum logic what have we learned? In Quantum Structures and the Nature of Reality (eds D Aerts, J Pykacz). no. 7 in Einstein Meets Magritte: An Interdisciplinary Reflection on Science, Nature, Art, Human Action and Society. pp. 1-36. Springer, The Netherlands.

24. Jauch JM. 1968 Foundations of quantum mechanics. Addison-Wesley series in advanced physics. Addison-Wesley Pub. Co, Reading, Massachusetts.

25. Bohm D. 1952 A suggested interpretation of the quantum theory in terms of 'hidden' variables. II. Phys. Rev. 85, 180-193. (doi:10.1103/PhysRev.85.180)

26. Schlosshauer M, Kofler J, Zeilinger A. 2013 A snapshot of foundational attitudes toward quantum mechanics. Stud. Hist. Phil. Sci. B 44, 222-230. (doi:10.1016/j.shpsb.2013.04.004)

27. Wigner E. 1932 On the quantum correction for thermodynamic equilibrium. Phys. Rev. 40, 749-759. (doi:10.1103/PhysRev.40.749) 
28. Cabello A, Estebaranz JM, Alcaine GC. 1996 Bell-Kochen-specker theorem: a proof with 18 vectors. Phys. Lett. A 212, 183-187. (doi:10.1016/0375-9601(96)00134-X)

29. Fine A. 1982 Joint distributions, quantum correlations, and commuting observables. J. Math. Phys. 23, 1306-1310. (doi:10.1063/1.525514)

30. Dzhafarov EN, Kujala JV. 2017 Contextuality-by-default 2.0: systems with binary random variables. In J. Acacio de Barros, Bob Coecke, and Emmanuel Pothos, editors, Quantum Interaction: 10th Int. Conf., QI 2016, volume 10106 of Lecture Notes in Computer Science. Springer International Publishing. (http://arxiv.org/abs/quant-ph/1604.04799).

31. de Barros JA, Kujala JV, Oas G. 2016 Negative probabilities and contextuality. J. Math. Psychol. 74, 34-45. (doi:10.1016/j.jmp.2016.04.014)

32. de Barros JA, Oas G. 2014 Negative probabilities and counter-factual reasoning in quantum cognition. Phys. Scr. T163, 014008. (doi:10.1088/0031-8949/2014/T163/014008)

33. Spekkens RW. 2008 Negativity and contextuality are equivalent notions of nonclassicality. Phys. Rev. Lett. 101, 020401. (doi:10.1103/PhysRevLett.101.020401)

34. de Barros JA, Suppes P. 2010 Probabilistic inequalities and upper probabilities in quantum mechanical entanglement. Manuscrito 33, 55-71.

35. Suppes P, Zanotti M. 1991 Existence of hidden variables having only upper probabilities. Found. Phys. 21, 1479-1499. (doi:10.1007/BF01889653)

36. Holik F, Saenz M, Plastino A. 2014 A discussion on the origin of quantum probabilities. Ann. Phys. 340, 293-310. (doi:10.1016/j.aop.2013.11.005)

37. Narens L. 2014 Probabilistic lattices: with applications to psychology. Singapore: World Scientific. Google-Books-ID: Bh23CgAAQBAJ

38. Khrennikov AY. 2009 Contextual approach to quantum formalism. New York, NY: Springer Science \& Business Media.

39. Werner RF. 1989 Quantum states with Einstein-Podolsky-Rosen correlations admitting a hidden-variable model. Phys. Rev. A 40, 4277-4281. (doi:10.1103/PhysRevA.40.4277)

40. Bohm D. 1952 A suggested interpretation of the quantum theory in terms of 'Hidden' variables. I. Phys. Rev. 85, 166-179. (doi:10.1103/PhysRev.85.166)

41. Plastino AR, Manzano D, Dehesa JS. 2009 Separability criteria and entanglement measures for pure states of N identical fermions. EPL 86, 20005. (doi:10.1209/0295-5075/86/20005)

42. Krause D, Sant'Anna AS, Volkov AG. 1999 Quasi-set theory for bosons and fermions: quantum distributions. Found. Phys. Lett. 12, 51-66. (doi:10.1023/A:1021678721611)

43. Krause D. 1992 On a quasi-set theory. Notre Dame J. Formal Logic 33, 402-411. (doi:10.1305/ ndjfl/1093634404) 